\title{
Obesity is associated with early hip fracture risk in postmenopausal women: a 25 -year follow-up
}

\author{
T. Rikkonen $^{1}$ (D) $\cdot$ R. Sund ${ }^{1} \cdot$ J. Sirola ${ }^{1,2} \cdot$ R. Honkanen ${ }^{1,3} \cdot$ K.E.S. Poole ${ }^{4} \cdot$ H. Kröger ${ }^{1,2}$
}

Received: 19 May 2020 / Accepted: 1 October 2020 / Published online: 23 October 2020

(C) The Author(s) 2020

\begin{abstract}
Summary Association of body mass index and hip fracture has been controversial. In this study, women with lowest and highest body weight had the highest fracture incidence. A 25 -year follow-up indicated that obesity associates with early hip fracture risk and suggested increasing trend in normal-weight women at a later stage.

Introduction Obesity is a pandemic health issue. Its association with hip fracture risk remains controversial. We studied the longterm relationship of body mass index and hip fracture incidence in postmenopausal women.

Methods The cohort of 12,715 Finnish women born in 1932-1941 was followed for 25 years, covering ages from 58 up to 83. Fractures and deaths were obtained from national registries. Women were investigated in deciles of BMI as well as in WHO weight categories (normal, overweight, or obese). The follow-up analysis was carried out in two age strata as "early" (5870 years) and "late" (> 70 years). Body weight information was updated accordingly. Femoral neck BMD was recorded for a subsample $(n=3163)$. Altogether, 427 hip fractures were observed.

Results A higher risk of early hip fracture was observed in obese and normal-weight compared with overweight women with hazard ratios (HRs) of $2.3((95 \% \mathrm{CI}) 1.4-3.7)$ and 2.0 (1.3-3.1) while no difference was observed in late hip fracture risk between the three WHO categories $(\log$ rank $p=0.14)$. All-cause mortality during the follow-up was $19.3 \%$. Compared with normal weight women, the obese women had a higher risk of death with an HR of 1.6 (1.4-1.8) and higher baseline BMD $(p<0.001)$. Faster bone loss was observed in the obese compared with other women $(p<0.001)$.

Conclusion Obesity associates with earlier hip fracture and higher postfracture mortality. The obese women with low BMD have clearly the highest risk of hip fracture. This combination increases hip fracture risk more than either of the factors alone. After 75 years of age, risk appears to increase more in normal weight women, but this trend is in need of further confirmation.
\end{abstract}

Keywords Aging $\cdot$ Body mass index $\cdot$ Bone mineral density $\cdot$ Follow-up study $\cdot$ General population $\cdot$ Hip fracture $\cdot$ Menopause $\cdot$ Obesity

Electronic supplementary material The online version of this article (https://doi.org/10.1007/s00198-020-05665-w) contains supplementary material, which is available to authorized users.

\section{T. Rikkonen}

toni.rikkonen@uef.fi

1 Kuopio Musculoskeletal Research Unit, University of Eastern Finland, Kuopio, Finland

2 Orthopaedics and Traumatology, Department of Orthopaedics, Traumatology and Hand Surgery, Kuopio University Hospital, Kuopio, Finland

3 Department of Psychiatry, University of Oulu, Oulu, Finland

4 University of Cambridge, Addenbrookes Hospital, Cambridge, UK

\section{Introduction}

Obesity is a major cause for preventable morbidity and premature mortality [1,2], while hip fracture has devastating consequences for independence and quality of life. Both obesity and hip fracture cause significant burden to health care and represent high societal costs $[3,4]$. Overall, the relationship between obesity, bone mass, and fracture risk in the aging population is a complex and rather contradicting topic depending on fracture type, population, age, and degree of overweight investigated. Recent studies suggest that the incidences of specific fracture types such as ankle and upper leg fractures were higher, while wrist and hip fracture was lower in obese compared with non-obese women [5]. These results also imply that factors such as lifestyle, exposure to daily activities, and comorbidities, rather than just body composition or bone 
mass, affect fracture risk. This is also supported by results where increasing levels of physical activity have been associated with higher incidence of wrist fracture, but lower incidence of hip fracture, in postmenopausal women $[6,7]$.

Up to a point, heavier individuals tend to have a higher BMD, but association between obesity and fracture risk is more complex. Key bone characteristics determining fracture risk appear to scale better in proportion of lean, rather than fat or total body mass [8]. This demonstrates the biomechanical nature of bones adapting to muscle contraction loads rather than sheer body weight itself. In obese women, the proportion of lean mass becomes an even smaller fraction of total body weight, further deteriorating this relative association. In case of a fall, heavier body weight also increases impact forces leading to injury which may have an accumulative effect on certain fracture types. However, body weight may also associate with protective factors such as increased subcutaneous padding and non-protective such as slower responses to falling or different direction of falls [9].

The joint effects of age, health behavior, comorbidities, and other competing events, such as higher mortality, are overlapping with obesity. Some health disorders commonly seen with obesity, such as type 2 diabetes (T2DM), seem to increase fracture risk at the hip [10], proximal humerus, ankle, and upper leg $[11,12]$. Paradoxically, obesity and T2DM also have a positive association with BMD [13,14]. This example of a seeming conflict makes fracture risk estimation with BMD alone prone to underestimation. It is challenging to make any conclusive comparison of these findings. While it has been shown that several clinical factors in addition to BMD or BMI can independently predict fracture risk, very few longterm follow-up studies focusing on obesity from the late middle age up to old age have been conducted $[15,16]$. It is uncertain how obesity predicts postmenopausal hip fracture, its type, and postfracture mortality. Our objective was to focus on the role of obesity in the occurrence of postmenopausal hip fractures in a long-term prospective cohort study covering up to 25 years.

\section{Materials and methods}

The study population consisted of the ongoing Kuopio Osteoporosis Risk Factor and Prevention (OSTPRE) study cohort. This population-based long-term follow-up study includes all the 14,220 Caucasian women born between 1932 and 1941 who lived in the Kuopio Province, Eastern Finland in April 1989. A postal questionnaire was mailed to 14,120 of these women at baseline in 1989 with 13,100 (92.8\%) responding. The follow-up questionnaires were mailed at 5year intervals $(1994,1999,2004,2009$, and 2014) to women who responded to the baseline enquiry and were alive at the time. The response rate varied between $93 \%$ and $80 \%$ throughout the study. The OSTPRE study was approved by the Kuopio University Hospital Ethics Committee on 28
October 1986 and is performed in accordance with the ethical standards of the Declaration of Helsinki. Informed consent has been provided before the onset of each data collection.

\section{Follow-up and baseline age}

The final study sample $(n=12,715)$ consisted of women with available height and weight information. Due to the 10-year age range, women's initial follow-up age in 1989 varied from 47 to 57 years. Therefore, start of the follow-up was age matched according to women's 58th birthday by using either the baseline, 5th, or 10th year questionnaire information, whichever closest. Correspondingly, the start of register follow-up period was also matched at the 58-year birthday for both outcomes of interest (hip fracture and death). The individual baseline day for the analysis was set from the oldest to youngest women between 1 January 1990 and 31 December 1999, respectively. Follow-up was terminated to end of the registry data (31 December 2014) or to the event of interest (hip fracture and death) whichever occurred first. During the follow-up analysis, women reached a mean (median) age of 77.9 (77.8) years over a follow-up time of 18.3 (18.7) years. Hip fracture risk was investigated in two separate age periods as early (58-70 years) and late ( $>70$ years) due to non-proportional hazard between the groups during the first half of the follow-up.

\section{Bone mineral density measurements}

Baseline responders were asked about their willingness to participate in bone densitometry (DXA) and clinical measurement protocols. Altogether, 11,055 women stated their willingness, which formed a pool for a stratified random sample of 3686 women invited to the measurements. Out of these, 3222 women underwent the baseline DXA scan. This sample consisted of a random population sample $(n=2025)$ and $100 \%$ samples $(n=1197)$ of women with higher risk profiles, including menopause within 2 years, diseases, or medication affecting bone, such as rheumatoid arthritis [17]. The DXA subsample used in this study $(n=3163)$ consisted of women with valid femoral neck bone densitometry at baseline 1989, at 5 th year or at 10th year follow-up, where closest matching to 58 years of age was used as a baseline value. Subjects with both baseline and 25-year BMD follow-up data available $(n=$ 792) formed the subsample for proportional bone loss rate (\%) comparison between groups. Body composition follow-up data was not recorded at the time being. Femoral neck T-score value was determined according to the Third National Health and Nutrition Examination Survey (NHANES III) with reference to white women aged 20-29 years [18].

The BMD measurements were carried out using a Lunar DPX scanner (Lunar, Madison, WI, USA) at both baseline (1989) and 5th year follow-up (1994). The original scanner was replaced with a DPX-IQ during the 10 th year 
measurement in 1999. The manufacturer's quality protocol was followed daily. To reveal the agreement between the scanners, 90 women were scanned with both instruments on the same day. The use of patient measurements rather than phantom measurements was emphasized. The long-term reproducibility of the DXA scanner determined by phantom measurements was $0.4 \%$ [1]. The detailed description of OSTPRE clinical measurement follow-up protocol and DXA cross-calibration has been published previously [6].

\section{Body mass index}

Body mass index was calculated from self-reported height and weight. The self-reported weight and measured weight have previously shown a good level of agreement in postmenopausal women and are considered reasonably reliable for BMI calculations [19]. In this cohort, the baseline body weight of the DXA subsample $(n=3163)$ showed a high correlation (Pearson $r=0.97)$ between the self-reported $(67.8 \mathrm{~kg}$ (SD $11.7)$ ) and measured baseline values (68.8 $\mathrm{kg}$ (SD 11.9) which persisted throughout the follow-up.

The BMI was categorized in two ways: first according to the WHO definition $\left(\mathrm{kg} / \mathrm{m}^{2}\right)$ as normal weight (18.5-24.9), overweight (25.0-29.9), and obese (30.0 or higher) and second, BMI was modeled in deciles to represent the overall 25year hip fracture incidence. The hip fracture probability associated with a given BMI level was calculated by dividing the total number of hip fractures by the attributable follow-up time in person years for each BMI decile.

Available weight and height information closest to the age of 58 years were used as a baseline value to investigate BMI and early hip fracture incidence between 58 and 70 years. Correspondingly, self-reported weight at 70 years of age was utilized to update the BMI information for late hip fracture analysis. Both BMI values were determined by utilizing available follow-up information (baseline, 5th, 10th, 15th, 20th) whichever was closest to the age of interest. The correlation of baseline weight against the 5 th and 10th year values was 0.92 and 0.87 with a mean weight increase of $+2.2 \mathrm{~kg}$ ((SD) 4.9 ) and $+3.3 \mathrm{~kg}(6.1)$ towards the latter, respectively. A small proportion of women with low BMI $(15.0-18.49, n=59)$ were excluded from the normal-weight category in the WHO classification but are included to the lowest decile of BMI presenting the overall incidence rate of hip fractures in the 25-year follow-up.

\section{Registry outcomes: fractures, causes of fracture, and deaths}

The cohort's hip fracture information was obtained from the nationwide Hospital Discharge Register data (HILMO) as well as by postal enquiries sent to the participants (at 10, 15, 20, and 25 follow-up years). All self-reported hip fractures during the years 1989-2014 were validated by crosschecking patient records. The Finnish Hospital Discharge Register has previously been shown to reliably identify virtually all hip fractures [20]. The relevant International Classification of Diseases (ICD) codes were used to include femoral neck, pertrochanteric, and subtrochanteric fractures (ICD-10 S72.0-S72.2). Women with pathologic (e.g., metastasis) and periprosthetic hip fractures were excluded. We have previously shown that the number of hip fractures from the register was significantly higher than the self-reported one. Women who did not respond to the OSTPRE survey were shown to have significantly higher hip fracture incidence. A study based solely on self-reported hip fractures would have resulted in biased risk estimates. Our previous validation study showed self-reports lacking $38 \%$ of hip fractures in this long-term follow-up [21].

External causes of hip fracture were categorized according to the Finnish version of ICD-9 that was in use between 1987 and 1995 (E800-E999), and ICD-10 was used since 1996 (V01.0-Y96.9) into low- and high-energy categories. Fractures due to road traffic crashes resulting in motor vehicle, bicycle, or pedestrian injuries or due to a fall from a height of $1 \mathrm{~m}$ or over were regarded as high-energy trauma (ICD-9; E803E, E882A, and ICD-10; V01-V89, W10, W11, W17, W22). Fractures related to a fall on level, slip fall, or falling from a height of less than $1 \mathrm{~m}$ were considered low-energy trauma (ICD-9; E881A, E883A, E889A, and ICD-10; W00W06, W19). Events with missing or unspecified information of trauma energy involved (such as stress fracture, intoxication, fire, complication) were categorized as unclear (ICD-9; E929X, E979A, and ICD-10; X50, X57, XX58, X59, Y83).

Causes of death were recorded by a certified physician according to the national adaptation of the ICD. The hip fracture risk analysis was stopped at the end of available information on time and cause of death from the National Causes of Death Register (31 December 2014), which have previously been shown to be accurate [22]. Survival analyses were carried out based on all-cause mortality.

\section{Statistical analyses}

The 58th birthday formed the baseline for the analysis. Depending on the outcome of interest, follow-up was terminated on the date of death, hip fracture, or at the end of the registry period in 31 December 2014. Overall, hip fracture risk for normal, overweight, and obese groups was estimated by using Kaplan-Meier estimation with a mean (median) followup time of 18.5 (19.0) years, 18.4 (18.8) years, and 17.7 (18.0) years, respectively. Since proportional hazard assumption of obese women was not met across the whole age, we split the follow-up data in two age strata: from 58 to 70 years as "early" and over 70 years as "late." Cox proportional hazard regression model was used to estimate hazard ratios with their 
respective $95 \% \mathrm{CI}$ in two follow-up sections. By using timedependent covariate analysis in Cox regression, a nonproportional hazard was observed between the study groups hip fracture rate during the first follow-up section from 58 to 70 years of age $(p<0.001)$. Difference between baseline characteristics, distribution of fracture type, and trauma energy were investigated using $t$ test and chi-squared methods. Overweight group was considered the reference for the primary analysis due to lowest incidence rate in hip fracture outcomes, while normal weight group was the reference group in the mortality analyses.

\section{Results}

\section{Body mass index}

The cohort consisted of 12,715 women with a mean (median) follow-up time of 18.3 (18.7) years. The mean baseline BMI was $26.7 \mathrm{~kg} / \mathrm{m}^{2}$, where $39.6 \%(n=5039)$ of the women had normal body weight. Altogether, $0.5 \%(n=59)$ of women were underweight (BMI of 15.0-18.49) at baseline with five hip fractures during the follow-up. At the age of 70 years, the number of underweight women was 131 . These women were excluded from the normal-weight WHO category but are included within the lowest BMI decile showing overall hip fracture incidence. Corresponding proportions of overweight $(n=$ $5082)$ and obese $(n=2535)$ women at baseline were $39.9 \%$ and $19.9 \%$, respectively (Table 1). Overall, lower weight gain during the first 10 years was observed in the normal BMI group ((mean, SD) $+2.5 \mathrm{~kg}, 4.7)$ ) compared with overweight $(+3.2 \mathrm{~kg}, 5.7)$ and obese $(+3.4 \mathrm{~kg}, 8.6)$ women (ANOVA $p<0.001)$. Mean weight change according to the baseline category did not show significant changes within groups during the total follow-up as shown in Fig. 5 (Appendix). The proportion (\%) of observed crossover between BMI categories according to the body weight set at 58 and 70 years of age are presented in Table 2 (Appendix).

\section{Hip fracture risk and mortality}

Total follow-up time was 233,096 person-years, during which 427 women had a hip fracture with a crude incidence of 24.4 per 10,000 person years and a prevalence of $3.4 \%$ at the end of

Table 1 Baseline characteristics of the total study population $(N=12,715)$ and according to the WHO definition $\left(\mathrm{kg} / \mathrm{m}^{2}\right)$ as underweight $(15.0-18.49)$, normal weight (18.5-24.9), overweight (25.0-29.9), and obese (30.0 or higher) with their respective mean (SD) or proportion (\%)

\begin{tabular}{|c|c|c|c|c|c|c|}
\hline Baseline characteristic & $\begin{array}{l}\text { Total } 100 \% \\
(N=12,715)\end{array}$ & $\begin{array}{l}\text { Underweight } 0.5 \% \\
(n=59)\end{array}$ & $\begin{array}{l}\text { Normal } 39.6 \% \\
(n=5039)\end{array}$ & $\begin{array}{l}\text { Overweight } 40.0 \% \\
(n=5082)\end{array}$ & $\begin{array}{l}\text { Obese } 19.9 \% \\
(n=2535)\end{array}$ & $\begin{array}{l}p \\
\text { value }^{\mathrm{a}}\end{array}$ \\
\hline Age (year) & $58.0(0)$ & $58.0(0)$ & $58.0(0)$ & $58.0(0)$ & $58.0(0)$ & ns \\
\hline Height (cm) & $161.1(0.5)$ & $162.4(6.3)$ & $161.4(5.3)$ & $161.1(5.2)$ & $160.8(5.3)$ & $<0.001$ \\
\hline Weight (kg) & $68.3(11.9)$ & $47.8(5.6)$ & $59.3(5.7)$ & $69.4(6.4)$ & $84.5(11.2)$ & $<0.001$ \\
\hline BMI $\left(\mathrm{kg} / \mathrm{m}^{2}\right)$ & $26.7(4.5)$ & $17.7(0.8)$ & $22.9(1.5)$ & $27.2(1.4)$ & $33.6(38)$ & $<0.001$ \\
\hline Current smoker (\%) & 25.8 & 40.7 & 26.2 & 25.4 & 24.7 & $<0.05$ \\
\hline Postmenopausal (\%) & 93.4 & 93.2 & 93.6 & 93.4 & 93.0 & $\mathrm{~ns}$ \\
\hline Hip fracture, $n(\%)$ & 427 & 5 & 173 & 157 & 92 & \\
\hline Femoral neck & $296(69.3)$ & $3(60.0)$ & $126(78.2)$ & $104(66.2)$ & $63(68.5)$ & ns \\
\hline Pertrochanteric & $106(24.8)$ & $2(40.0)$ & $38(22.0)$ & $44(28.0)$ & $22(23.9)$ & $\mathrm{ns}$ \\
\hline Subtrochanteric & $25(5.9)$ & 0 & $9(5.2)$ & $9(5.7)$ & $7(7.6)$ & ns \\
\hline \multicolumn{7}{|l|}{ Fracture energy, $n(\%)$} \\
\hline Unclear & $54(12.6)$ & & $26(14.6)$ & $15(9.6)$ & $13(14.1)$ & ns \\
\hline Low & $341(79.9)$ & $5(100)$ & $140(78.7)$ & $129(82.2)$ & $72(78.3)$ & ns \\
\hline High & $32(7.5)$ & & $12(6.7)$ & $13(8.3)$ & $7(7.6)$ & ns \\
\hline Subsample $^{\mathrm{b}}$ & $n=3163$ & $n=12$ & $n=1279$ & $n=1256$ & $n=616$ & \\
\hline $\begin{array}{l}\text { Mean femoral neck BMD } \\
\left(\mathrm{g} / \mathrm{cm}^{2}\right)^{\mathrm{b}}\end{array}$ & $0.914(0.127)$ & $0.777(0.102)$ & $0.867(0.119)$ & $0.929(0.118)$ & $0.985(0.122)$ & $<0.001$ \\
\hline $\begin{array}{l}\text { Mean femoral neck T-score } \\
\text { (NHANES III) }^{\text {b }}\end{array}$ & 0.45 & -0.69 & 0.06 & 0.58 & 1.04 & $<0.001$ \\
\hline $\begin{array}{l}\text { Mean bone loss in } 25 \text { years } \\
(\%)^{\text {b,c }}\end{array}$ & -8.7 & -4.8 & -7.7 & -9.1 & -10.6 & $<0.01$ \\
\hline
\end{tabular}

${ }^{a}$ Difference between groups ( $t$ test and chi-squared)

${ }^{\mathrm{b}}$ Subjects with available baseline BMD data $(n=3163)$. Femoral neck BMD values and T-score according to NHANES III white women reference

${ }^{\mathrm{c}}$ Subjects with 25-year BMD follow-up data available $(n=792)$ in underweight $(n=3)$, normal $(n=350)$, overweight $(n=319)$, and obese $(n=120)$ groups 
the follow-up. The mean age at the time of hip fracture during the study follow-up was 72.6 years (range 58.3-82.5). The number of hip fractures and occurrence (\%) among normal, overweight, and obese women during the whole follow-up were $173(3.4 \%), 157(3.1 \%)$, and $92(3.6 \%)$. Higher mortality in obese women affecting hip fracture risk as a competing outcome was present. Regardless, their overall hip fracture incidence rate was higher compared with overweight and normal-weight categories during the follow-up with respective values of 18.5 ((95\% CI $15.9-21.4)), 16.8$ (14.3-19.6), and 20.5 (16.6-25.0) per 10,000 person years.

Overall, significantly higher hip fracture risk was observed in the obese and normal-weight groups compared with overweight women during the first half of the follow-up before 70 years of age (Fig. 1). This early hip fracture risk in obese and normal-weight women had hazard ratios (HRs) of 2.3 $((95 \% \mathrm{CI}) 1.4-3.7, p<0.01)$ and $2.0(1.3-3.1, p<0.01)$ compared with overweight category. During the latter half of the follow-up ( $>70$ years), no difference (Kaplan-Meier curves, log-rank, $p=0.14$ ) was seen in fracture risk between the categories (Fig. 2). The overall incidence rate of hip fractures in 25 years according to BMI deciles suggested a non-linear association between the two (Fig. 3).

Obese women were more prone to suffer a hip fracture at younger age, while the respective age gap narrowed with normal-weight category during aging. With a hip fracture probability of $1 \%, 2 \%$, and $4 \%$, the observed age gap between the lowest (overweight) and highest (obese) categories was $5.1,2.1$, and 1.3 years, respectively. In practice, obese women reached their $1 \%$ probability at the age of 66.7 years of age, while the respective age for the overweight group was 71.8 .
The categories with their respective fracture probabilities and ages are shown in Table 3 Appendix.

The highest all-cause mortality during the follow-up was observed in obese women $(25.5 \%, n=646)$, followed by the overweight $(18.3 \%, n=930)$ and normal-weight $(17.2 \%, n=$ 876) categories. Overall, the respective age-adjusted hazard ratio for death in obese women was $1.6((95 \% \mathrm{CI}) 1.4-1.8)$ compared with normal-weight women which had the lowest hazard during the follow-up.

\section{Fracture type and postfracture mortality}

No difference was observed in distribution of hip fracture types among femoral neck (69.3\%), pertrochanteric (24.8\%), and subtrochanteric (5.9\%) regions between the WHO BMI groups. Out of 427 women who sustained a hip fracture, the overall mortality during follow-up was $41 \%$ (175), whereas $121(28.3 \%)$ of the women died within 5 years after the incident. Survival analysis showed a tendency towards a higher 5year postfracture mortality after hip fracture in the obese compared with overweight women when adjusted for age at the time of fracture, with a HR of $1.5((95 \% \mathrm{CI}) 0.9-2.4, p=0.1)$ which, however, did not reach statistical significance in this cohort.

\section{Bone density}

A stratified random sample of 3163 women available with DXA data formed the subsample for additional risk analysis with a mean BMD femoral neck T-score of +0.45 according to NHANES III reference. Increasing BMD value $\left(\mathrm{g} / \mathrm{cm}^{2}\right)$ and
Fig. 1 Cumulative probability of "early" hip fracture at ages 58-70 according to baseline BMI for normal (green), overweight (orange), and obese (red) women (Kaplan-Meier curves, log-rank, $p<0.01)$

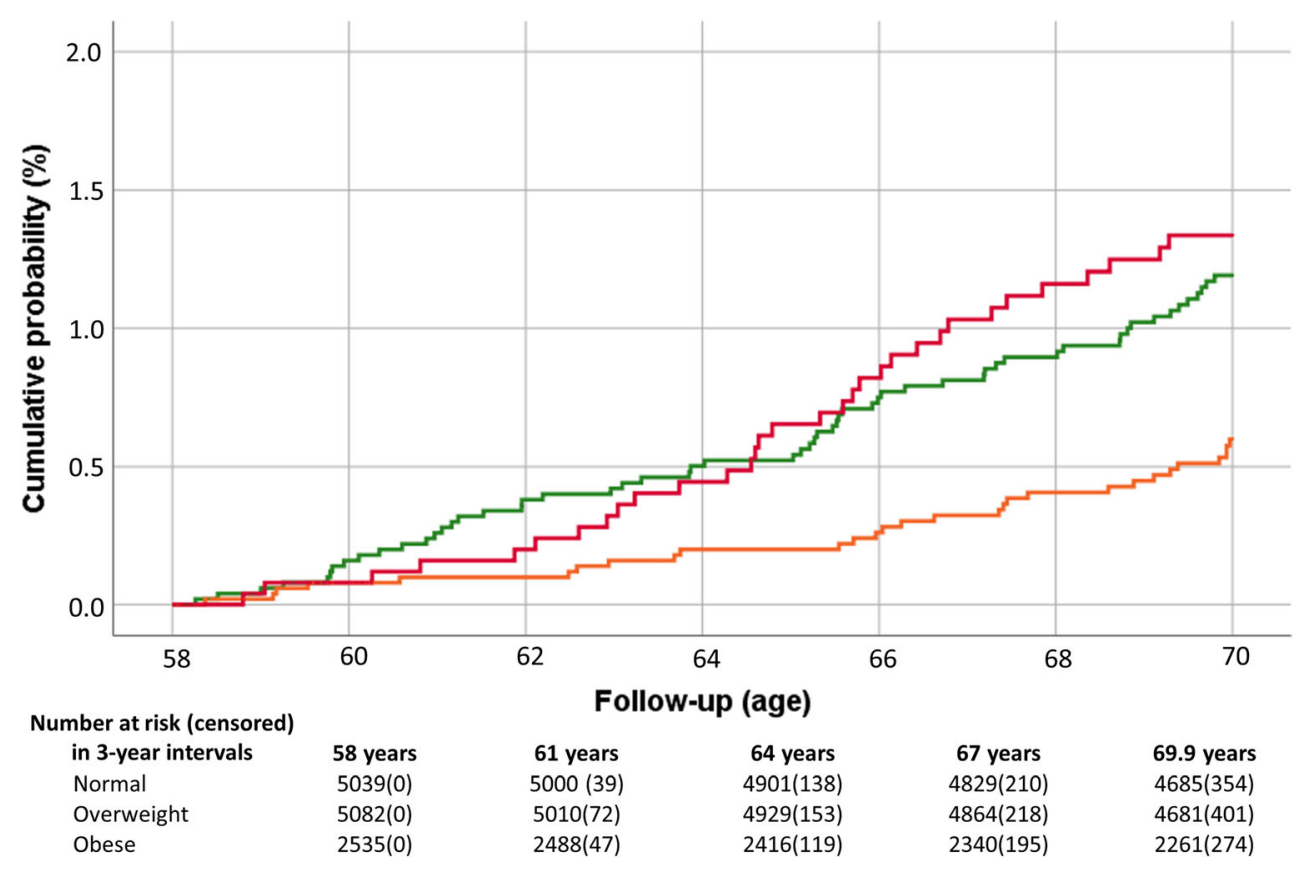


Fig. 2 Cumulative probability of "late" hip fracture at ages 70-83 according to baseline BMI for normal (green), overweight (orange), and obese (red) women (Kaplan-Meier curves, log-rank, $p=0.14)$

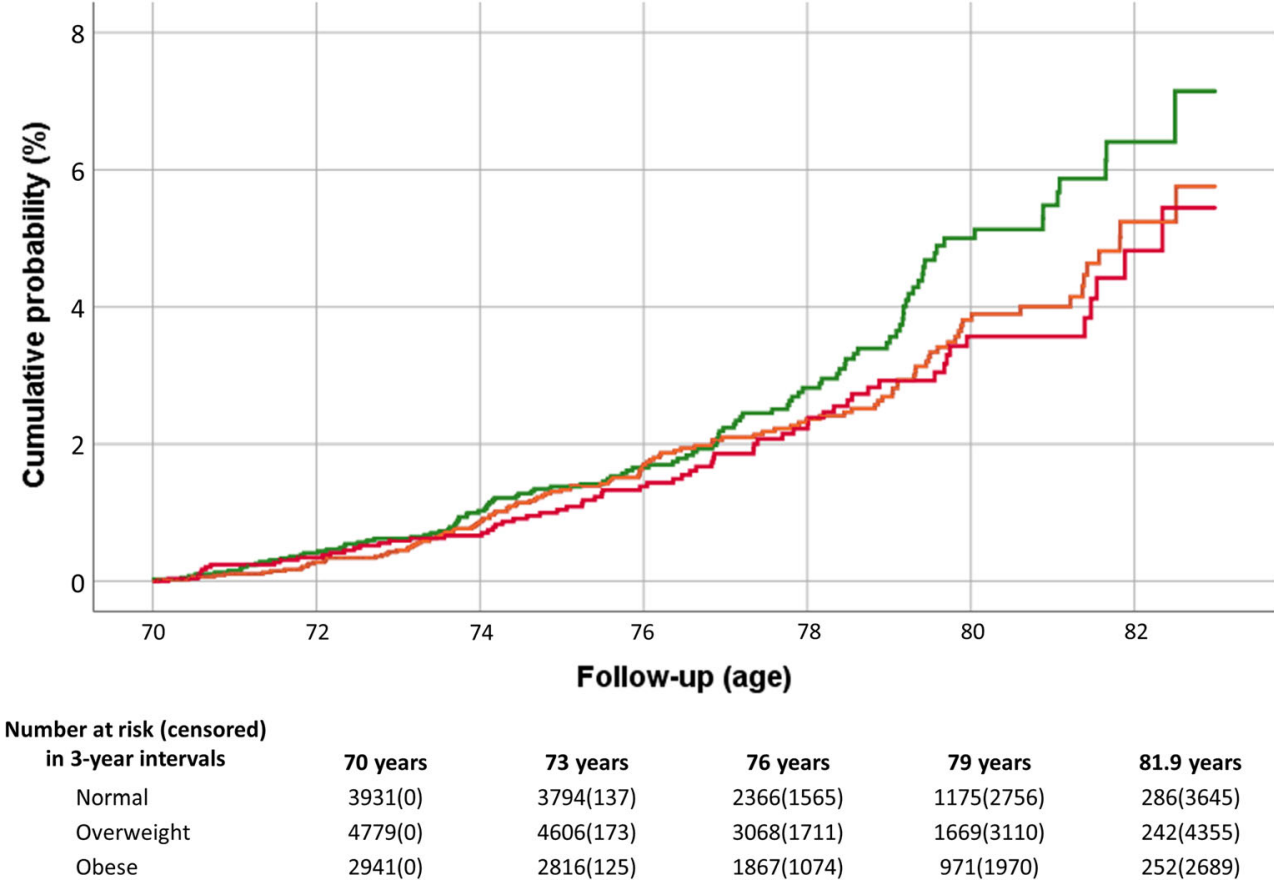

T-score was observed between normal, overweight, and obese weight categories, with mean values of 0.866 (T-score 0.05 ), 0.929 (0.58), and 0.985 (1.04), respectively (ANOVA, $p<0.001)$. The number of OSTPRE women with osteoporosis at the baseline is previously known to be low since NHANES III BMD cutoff values are lower than Finnish national values, with a corresponding number of osteoporotic $(n=4)$, osteopenic $(n=254)$, or normal $(n=2905)$ women
[18]. Using national (Finnish) cutoff values, the proportion would still have remained low with corresponding numbers of 74, 524, and 2565 women, respectively [23]. However, prevalent overweight in this cohort contributes to slightly elevated mean T-score. Overall, obese women belonging to the lowest BMD tertile showed the highest hip fracture incidence with a rate of $58.6(27.2-111.2)$ per 10,000 person years (Fig. 4).
Fig. 3 Cumulative number and incidence rate of hip fractures (per 10,000 person years) in 25 years according to baseline BMI percentile. The highest and lowest deciles are presented in groups of $5 \%$

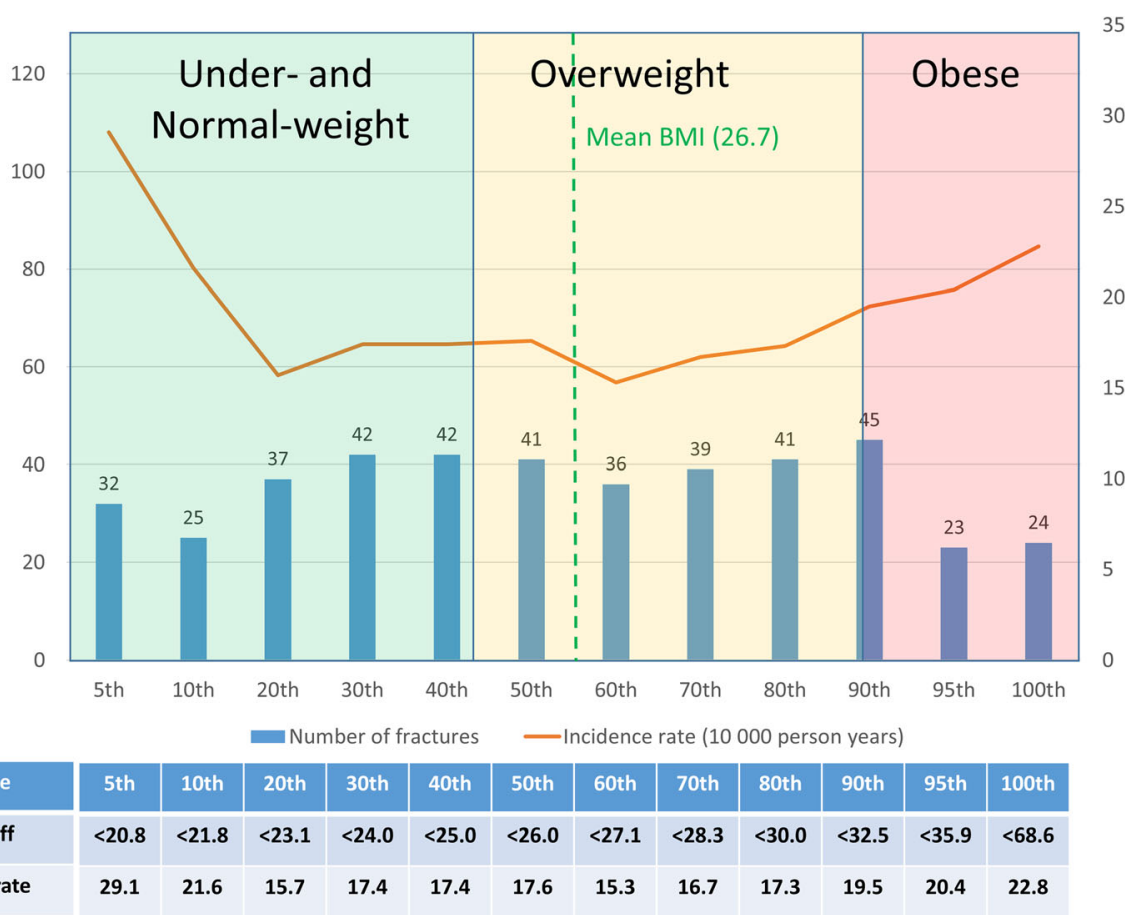




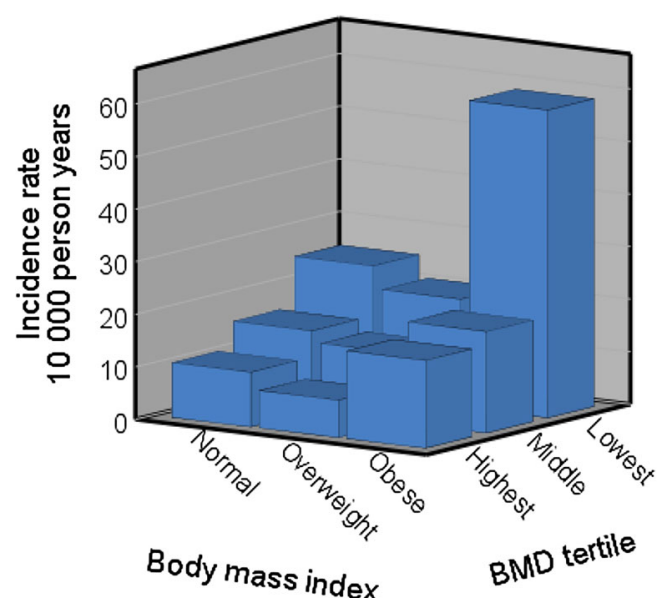

Fig. 4 A 25-year hip fracture incidence rate in a DXA subsample of 3163 women with a total of 100 hip fractures according to baseline BMI category and BMD tertile. Cutoff values for BMD tertile are $0.855 \mathrm{~g} / \mathrm{cm}^{2}$ and $0.963 \mathrm{~g} / \mathrm{cm}^{2}$

\section{Discussion}

This prospective cohort study showed an association between obesity and higher hip fracture incidence before 70 years of age in postmenopausal women. Higher all-cause mortality in obese women was a competing risk for hip fracture. At 70 years of age, obesity did not seem to be associated with higher hip fracture rate in this cohort, whereas normal BMI showed a tendency for faster increase in fracture rate after around 77 years of age. Altogether, during the follow-up period of 25 years, the highest age-matched hip fracture rate was seen in the lowest 5 th percentile of BMI, followed by the most obese 95th percentile of women. This relationship between hip fracture incidence and BMI resembles a non-linear association, where the leanest and most obese women share the highest overall risk but at a different age.

The result is consistent with some of the previous findings where either low or high BMI has been reported as a risk factor for fractures $[24,25]$. However, the association of BMI and hip fracture also varies with age which might explain some of the contradictive observations between BMI and fracture risk in studies with relatively short follow-up, wide age range, or both. In this study, light body weight and obesity both increased fracture risk, suggesting that BMI follows a non-linear relationship with hip fracture risk in postmenopausal women and that the association is age specific. Thus, obesity is associated with early hip fracture risk which is surpassed by lean women at around 75 years of age.

Additional factors including hip fracture type, trauma energy, and 5-year postfracture mortality were also investigated in BMI categories and deciles to reveal associations that might explain previous obesity-related contradictions in hip fractures. However, hip fracture by anatomical region of femoral neck, pertrochanteric, and subtrochanteric types was not associated with BMI. In addition, the trauma energy involved appeared to be similar throughout the body weight distribution. A tendency towards higher age-adjusted 5-year postfracture mortality after hip fracture was detected in obese women. Although this was approximately the same as the all-cause mortality in obese women. Thus, a hip fracture event does not appear to bring an additional effect on the already higher mortality risk among the obese women compared with others.

The lowest fracture risk was seen in the overweight women. Previous studies have suggested that higher BMI modifies the fracture risk at least through increased BMD due to somewhat greater skeletal loading. It also translates to higher impact forces when falling and may offer greater impact absorption by subcutaneous soft tissue. In addition, muscle mass and strength relate to BMI and affect bone strength. However, difference in muscle mass explaining BMI variance is not expected to play a significant role in this age range. Muscle strength and lean mass should be considered separately since both independently associate with postmenopausal BMD in this cohort [26]. Subtle overweight in elderly is also shown to associate with lower risk in other health outcomes such as incidence of fatal cardiovascular event and slightly lower mortality rate [27]. Thus, lower hip fracture risk in overweight group may have similar trend with these outcomes that are probably explained by factors such as lifestyle, nutrition, and overall health.

In this study, obesity associated with both early hip fracture risk and higher all-cause mortality. The impact of BMI on hip fracture risk in the aging population is a complex and multifactorial topic. Numerous overlapping risk factors and competing events such as comorbidities, poor health behavior, low functional capacity, and excessive all-cause mortality commonly coexist with obesity [28-31]. Variation in these factors alone produces a significant bias for fracture risk estimation. Mortality in general is suggested to have stronger association with BMI. Being overweight or obese in young adulthood appears to increase this risk, as well as being underweight or obese in later life [32]. Similar association between mortality with low and high BMI in later life also aligns with the current observation of hip fracture incidence. Altogether, the results indicate a low hip fracture probability in overweight women compared with normal and obese categories. A similar reduction associated with overweight women has also been reported in all-cause mortality [33]. Obesity-related excess mortality is shown to decline with age [34] which can also be observed with the hip fracture probability.

The current study consists of a very long follow-up of a homogenous sample of postmenopausal women. Living in the same area (Kuopio province) with a rather narrow age range (of 10 years) makes these findings better controlled but more cohort specific. The mean hip fracture age is limited by the follow-up duration. Thus, it is not comparable with population mean age in hip fracture incidence, where limiting factors such as follow-up duration, gender, or age are not present. 
Previously, several large cohort studies and meta-analyses have studied women's BMI and osteoporotic fractures in age ranges from 20 up to +100 years. The results have been somewhat contradictory mainly suggesting that obesity might even be associated with a lower hip fracture risk but may promote some other osteoporotic fracture types which are mediated partly through BMD [12, 35-37]. However, methodological methodological variations such as study settings, type of fractures, follow-up times, heterogeneity of study populations, and age ranges make the overall association inconclusive. In addition, the follow-up times have commonly been limited to well under 10 years which makes any age and weight changespecific projections problematic. This study aimed to investigate long-term change in BMI and fracture risk while controlling for the same chronological age throughout the follow-up.

The baseline BMI and bone mass were determined according to time at the age of 58 and updated at 70 years of age. This approach was likely to set off the time between the most recent weight information available and the baseline date up to a maximum of 2.5 years. Although no significant change in BMD or BMI within this period was detected, the bias cannot be totally excluded. However, it is very unlikely that the observed mean change of $1 \mathrm{~kg}$ in body weight during any 2.5 -year period would cause systematic error resulting in biased hip fracture or mortality estimates. In addition, the longitudinal BMD data was only available for small subsample, which prevented more robust statistical analyses controlling for possible interaction between bone parameters and BMI during the follow-up.

In conclusion, obesity associates with early hip fracture risk in postmenopausal women before 70 years of age. The risk is increased in normal-weight women at a later stage. Thus, body mass index shows a non-linear relationship with hip fracture in postmenopausal women. Obese women with low BMD have even higher probability of hip fracture than with either of these risk factors alone. A tendency for a higher post hip fracture mortality in obese women aligns with their higher allcause mortality. Obesity should be considered as a risk factor for early hip fracture as well as mortality.

Funding Open access funding provided by University of Eastern Finland (UEF) including Kuopio University Hospital. This study has been financially supported by the Juho Vainio Foundation, Finnish Academy (Grant No. 310831/2017), Finnish Cultural Foundation (The North Savo Regional Fund), and Sigrid Juselius Foundation.

Data availability Not applicable.

\section{Compliance with ethical standards}

\section{Conflicts of interest None.}

Ethics approval All procedures performed in studies involving human participants were in accordance with the ethical standards of the Kuopio
University Hospital ethics committee and with the 1964 Helsinki declaration and its later amendments.

Consent to participate Informed consent was obtained from all individual participants included in the study.

Consent for publication The informed consent has been reviewed and granted by all participants to utilize the study data for the scientific publications.

Code availability Not applicable.

Open Access This article is licensed under a Creative Commons Attribution-NonCommercial 4.0 International License, which permits any non-commercial use, sharing, adaptation, distribution and reproduction in any medium or format, as long as you give appropriate credit to the original author(s) and the source, provide a link to the Creative Commons licence, and indicate if changes were made. The images or other third party material in this article are included in the article's Creative Commons licence, unless indicated otherwise in a credit line to the material. If material is not included in the article's Creative Commons licence and your intended use is not permitted by statutory regulation or exceeds the permitted use, you will need to obtain permission directly from the copyright holder. To view a copy of this licence, visit http://creativecommons.org/licenses/by-nc/4.0/.

\section{References}

1. Mitchell S, Shaw D (2015) The worldwide epidemic of female obesity. Best Pract Res Clin Obstet Gynaecol 29(3):289-299

2. Calle EE, Rodriguez C, Walker-Thurmond K, Thun MJ (2003) Overweight, obesity, and mortality from cancer in a prospectively studied cohort of US adults. N Engl J Med 348(17):1625-1638

3. Braithwaite RS, Col NF, Wong JB (2003) Estimating hip fracture morbidity, mortality and costs. J Am Geriatr Soc 51(3):364-370

4. Withrow D, Alter DA (2011) The economic burden of obesity worldwide: a systematic review of the direct costs of obesity. Obes Rev 12(2):131-141

5. Compston JE, Watts NB, Chapurlat R, Cooper C, Boonen S, Greenspan S, Pfeilschifter J, Silverman S, Díez-Pérez A, Lindsay R, Saag KG, Netelenbos JC, Gehlbach S, Hooven FH, Flahive J, Adachi JD, Rossini M, Lacroix AZ, Roux C, Sambrook PN, Siris ES, Glow Investigators (2011) Obesity is not protective against fracture in postmenopausal women: GLOW. Am J Med 124(11): $1043-1050$

6. Rikkonen T, Salovaara K, Sirola J, Kärkkäinen M, Tuppurainen M, Jurvelin J, Honkanen R, Alhava E, Kröger H (2010) Physical activity slows femoral bone loss but promotes wrist fractures in postmenopausal women: a 15-year follow-up of the OSTPRE study. J Bone Miner Res 25(11):2332-2340

7. Feskanich D, Willett W, Colditz G (2002) Walking and leisure-time activity and risk of hip fracture in postmenopausal women. JAMA. 288(18):2300-2306

8. Beck TJ, Petit MA, Wu G, LeBoff MS, Cauley JA, Chen Z (2009) Does obesity really make the femur stronger? BMD, geometry, and fracture incidence in the women's health initiative-observational study. J Bone Miner Res 24(8):1369-1379

9. Crandall CJ, Yildiz VO, Wactawski-Wende J, Johnson KC, Chen Z, Going SB, Wright NC, Cauley JA (2015) Postmenopausal weight change and incidence of fracture: post hoc findings from women's health initiative observational study and clinical trials. BMJ. 350:h25 
10. Walsh JS, Vilaca T (2017) Obesity, type 2 diabetes and bone in adults. Calcif Tissue Int 100(5):528-535

11. De Liefde I, Van der Klift M, De Laet C, Van Daele P, Hofman A, Pols H (2005) Bone mineral density and fracture risk in type-2 diabetes mellitus: the Rotterdam study. Osteoporos Int 16(12): 1713-1720

12. De Laet C, Kanis J, Odén A et al (2005) Body mass index as a predictor of fracture risk: a meta-analysis. Osteoporos Int 16(11): $1330-1338$

13. Vestergaard P (2007) Discrepancies in bone mineral density and fracture risk in patients with type 1 and type 2 diabetes - a metaanalysis. Osteoporos Int 18(4):427-444

14. Felson DT, Zhang Y, Hannan MT, Anderson JJ (1993) Effects of weight and body mass index on bone mineral density in men and women: the Framingham study. J Bone Miner Res 8(5):567-573

15. Qazi S, Sirola J, Kröger H, Honkanen R, Isanejad M, Airaksinen O, Rikkonen T (2019) High postural sway is an independent risk factor for osteoporotic fractures but not for mortality in elderly women. $\mathrm{J}$ Bone Miner Res 34(5):817-824. https://doi.org/10.1002/jbmr.3664

16. Taylor BC, Schreiner PJ, Stone KL, Fink HA, Cummings SR, Nevitt MC, Bowman PJ, Ensrud KE (2004) Long-term prediction of incident hip fracture risk in elderly white women: study of osteoporotic fractures. J Am Geriatr Soc 52(9):1479-1486

17. Sirola J, Salovaara K, Tuppurainen M, Jurvelin J, Alhava E, Kröger H (2009) Sister's fracture history may be associated with perimenopausal bone fragility and modifies the predictability of fracture risk. Osteoporos Int 20(4):557-565

18. Looker AC, Orwoll ES, Johnston JRCC et al (1997) Prevalence of low femoral bone density in older US adults from NHANES III. J Bone Miner Res 12(11):1761-1768

19. Armstrong ME, Spencer EA, Cairns BJ et al (2011) Body mass index and physical activity in relation to the incidence of hip fracture in postmenopausal women. J Bone Miner Res 26(6):1330 1338

20. Sund R (2012) Quality of the finnish hospital discharge register: a systematic review. Scand J Public Health 40(6):505-515

21. Sund R, Honkanen R, Johansson H, Odén A, McCloskey E, Kanis J, Kröger H (2014) Evaluation of the FRAX model for hip fracture predictions in the population-based Kuopio osteoporosis risk factor and prevention study (OSTPRE). Calcif Tissue Int 95(1):39-45

22. Lahti RA, Penttilä A (2001) The validity of death certificates: routine validation of death certification and its effects on mortality statistics. Forensic Sci Int 115(1):15-32

23. Kröger H, Heikkinen J, Laitinen K, Kotaniemi A (1992) Dualenergy X-ray absorptiometry in normal women: a cross-sectional study of 717 finnish volunteers. Osteoporos Int 2(3):135-140

24. Holmberg AH, Johnell O, Nilsson PM, Nilsson J, Berglund G, Åkesson K (2006) Risk factors for fragility fracture in middle age. A prospective population-based study of 33,000 men and women. Osteoporos Int 17(7):1065-1077
25. Gnudi S, Sitta E, Lisi L (2009) Relationship of body mass index with main limb fragility fractures in postmenopausal women. J Bone Miner Metab 27(4):479-484

26. Rikkonen T, Sirola J, Salovaara K, Tuppurainen M, Jurvelin JS, Honkanen R, Kröger H (2012) Muscle strength and body composition are clinical indicators of osteoporosis. Calcif Tissue Int 91(2): $131-138$

27. Janssen I (2007) Morbidity and mortality risk associated with an overweight BMI in older men and women. Obesity. 15(7):18271840

28. Berrington de Gonzalez A, Hartge P, Cerhan JR et al (2010) Bodymass index and mortality among 1.46 million white adults. N Engl J Med 363(23):2211-2219

29. Calle EE, Thun MJ, Petrelli JM, Rodriguez C, Heath CW Jr (1999) Body-mass index and mortality in a prospective cohort of US adults. N Engl J Med 341(15):1097-1105

30. Bhaskaran K, dos Santos-Silva I, Leon DA, Douglas IJ, Smeeth L (2018) Association of BMI with overall and cause-specific mortality: a population-based cohort study of 3.6 million adults in the UK. Lancet Diabetes Endocrinol 6(12):944-953

31. Pataky Z, Armand S, Müller-Pinget S, Golay A, Allet L (2014) Effects of obesity on functional capacity. Obesity. 22(1):56-62

32. Corrada MM, Kawas CH, Mozaffar F, Paganini-Hill A (2006) Association of body mass index and weight change with all-cause mortality in the elderly. Am J Epidemiol 163(10):938-949

33. Flegal KM, Kit BK, Orpana H, Graubard BI (2013) Association of all-cause mortality with overweight and obesity using standard body mass index categories: a systematic review and meta-analysis. JAMA. 309(1):71-82

34. Bender R, Jöckel K, Trautner C, Spraul M, Berger M (1999) Effect of age on excess mortality in obesity. JAMA. 281(16):1498-1504

35. Johansson H, Kanis JA, Odén A, McCloskey E, Chapurlat RD, Christiansen C, Cummings SR, Diez-Perez A, Eisman JA, Fujiwara S, Glüer CC, Goltzman D, Hans D, Khaw KT, Krieg MA, Kröger H, LaCroix AZ, Lau E, Leslie WD, Mellström D, Melton LJ III, O'Neill TW, Pasco JA, Prior JC, Reid DM, Rivadeneira F, van Staa T, Yoshimura N, Zillikens MC (2014) A meta-analysis of the association of fracture risk and body mass index in women. J Bone Miner Res 29(1):223-233

36. Robbins J, Aragaki AK, Kooperberg C, Watts N, WactawskiWende J, Jackson RD, LeBoff MS, Lewis CE, Chen Z, Stefanick ML, Cauley J (2007) Factors associated with 5-year risk of hip fracture in postmenopausal women. JAMA. 298(20):2389-2398

37. Cummings SR, Nevitt MC, Browner WS, Stone K, Fox KM, Ensrud KE, Cauley J, Black D, Vogt TM (1995) Risk factors for hip fracture in white women. N Engl J Med 332(12):767-774

Publisher's note Springer Nature remains neutral with regard to jurisdictional claims in published maps and institutional affiliations. 